\title{
MODELLI DI URBANIZZAZIONE COSTIERA: MORFOLOGIA E COMPLESSITÀ STRUTTURALE, A SCALA URBANA E TERRITORIALE, NELLA REGIONE METROPOLITANA DI BARCELLONA ${ }^{1}$
}

\author{
Nicola Colaninno ${ }^{2}$ \\ Architetto \\ Josep Roca Cladera ${ }^{3}$ \\ Arquitecto
}

Remisión artículo: 13/05/2008
Centro de Política de Suelo y Valoraciones. Universitat Politècnica de Catalunya.

Keywords: Modelli di urbanizzazione, urbanizzazione costiera, interazione funzionale, protosistemi, territoriale, frammentazione, complessità, forma, cluster, costa mediterranea.

\section{Resumen.}

El trabajo se presenta como reflexión sobre la "forma" de diferentes tipologías de ciudades y acerca de las relaciones que las vinculan a un ámbito territorial, en una visión de desarrollo contemporáneo; con particular atención a la influencia que ejercita la cercanía al mar sobre los sistemas urbanos. El objetivo es delinear, gráficamente, esquemas de modelos a través de una operación de abstracción hecha sobre polígonos definidos por el proyecto CORINE Land Cover 2000; asignar, a cada esquema, valores numéricos a diferentes escalas, después de clasificar grupos distintos de asentamientos urbanos por medio de análisis estadísticos; reflexionar, finalmente, sobre los perímetros efectivos de interacción territorial entre los modelos definidos, puesto que, a lo largo de la costa mediterránea, los límites municipales se van difuminando a causa de las nuevas dinámicas urbanizadoras. Además se pretende estudiar conceptos como difusión y fragmentación urbana, "dibujando" dichos patrones de modelos de urbanización reconocibles sobre el territorio y estableciendo criterios de lectura lógico-conceptual de los fenómenos de desarrollo urbano.

\section{Summary.}

The work is presented as a reflection as a "form" of different cities typologies and proximity the relationships that bind a territorial basis, in a contemporary vision of development, with particular attention to the influence of urban systems close to the sea. The objective is to delineate, graphically schemes models through an operation of abstraction made on sites identified by Land Cover dataset of CORINE project for year 2000; allocate to each scheme, different scales of numerical values, after classifying different urban settlements groups by means of statistical analyses; showing, finally, on the effective perimeters the interaction between defined spatial models, because, along the Mediterranean coast, the municipal boundaries are blurring by the affect of the new dynamics developers. In addition seeks discussing concepts such as dissemination and urban fragmentation, "drawing" various patterns of recognizable urbanization models over the territory and establishing criteria for reading logical conceptual of the urban development phenomena.

\footnotetext{
${ }^{1}$ L'articolo è il prodotto della tesina di master in Gestión y Valoración Urbana, sostenuto nell'anno 2006/2007 presso la Universidad Politecnica de Cataluña, Barcellona, Spagna.

${ }^{2}$ Autore della tesi. Ricercatore presso il Centro de Política de Suelo y Valoraciones della Universidad Politecnica de Cataluña.

${ }^{3}$ Direttore della tesi. Cattedratico presso la Scuola Superiore di Architettura e direttore del Centro de Política de Suelo y Valoraciones della Universidad Politecnica de Cataluña.
}

Supporto tecnico: Carlos Marmolejo Durante; Montserrat Moix Bergadá. 


\section{Premessa}

Analizzando le città contemporanee si nota come lo spazio urbano si sia enormemente esteso, frammentato e disperso nel corso degli ultimi decenni; da città compatte si è passati ad insediamenti sempre più dilatati e diffusi sul territorio a causa di una crescita additiva dei tessuti urbani moderni e di dinamiche urbanizzatrici sempre più "modellanti" o forse "de-modellanti".

La stratificazione di epoche successive e delle fasi più rilevanti della crescita e delle trasformazioni delle città, dal preromano al medioevale, dal rinascimentale fino al contemporaneo e l'aumento demografico dovuto a processi di industrializzazione, con le conseguenti immigrazioni che provocano una moltiplicazione delle aree urbanizzate e la formazione delle attuali periferie, prodotto dall'epoca post-industriale, ci lasciano forme sempre diverse ma in qualche modo unite da una storia riconoscibile che funge da filo conduttore nel "disegno" di una città o di un'area metropolitana.

Ė possibile oggi osservare di frequente città sprawled, città "spalmate" sul territorio, che negli anni '60 del secolo passato hanno generato un punto di rottura con la consuetudine urbanistica europea, un punto di passaggio da una forma di città compatta, espressione della continuità formale, a quella dispersa dominata dal "segno" del frammento. L'immagine della città del passato si è perduta e lo spazio fisico resta oggi essenzialmente quello delle conurbazioni, mentre lo spazio funzionale è rappresentato dalle aree metropolitane, dalle regioni e dai sistemi urbani. Ciò che rimane è il territorio con le sue molteplici dimensioni, strutturali e sociali, che si concentrano in gruppi sempre più piccoli di nuclei edilizi od in aree urbane sempre meno identificabili; confini, gerarchie e interazioni sono cambiati ed è perciò necessario analizzare determinati fenomeni a differenti scale.

Gli effetti di tali fenomeni sono sicuramente osservabili oggi lungo le aree costiere dei paesi europei, dove gran parte delle concentrazioni urbane tende a svilupparsi in maniera sempre più massiva e continua, con una forte "pressione urbanizzatrice" (Figura 1); in determinate stagioni ed in determinate ubicazioni inoltre, i flussi migratori umani tendono ad incrementare in modo considerevole tali dinamiche urbane.

Figura 1. Visione notturna dell' Europa.

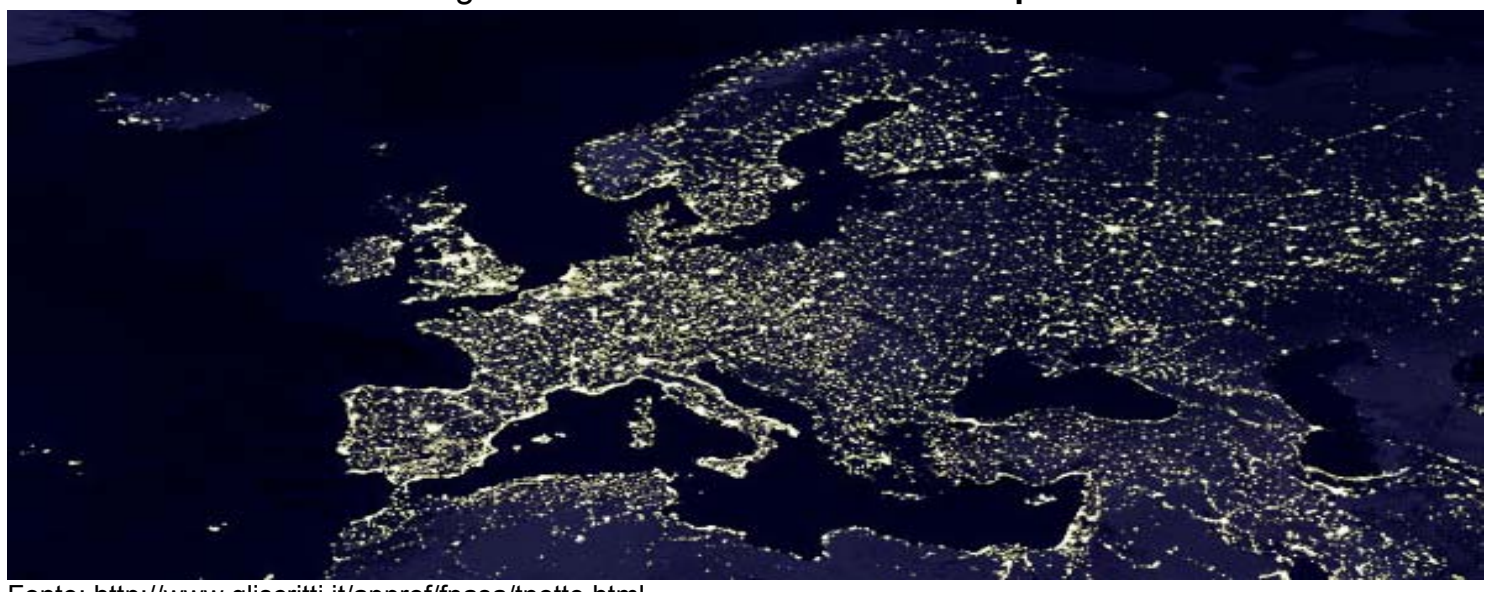

Fonte: http://www.gliscritti.it/approf/fnasa/tnotte.html

La dinamica demografica, in atto lungo le coste dei Paesi mediterranei, ad esempio, con la complicità di una pressione turistica importante, mostra chiaramente i segni di un "carico umano" in crescita, che è alla base dei fenomeni di dispersione e dilatamento sul territorio, poiché comporta una forte espansione dell'edificato, sia residenziale che di servizio: prime e seconde case, ricettività alberghiera, vie e nodi di comunicazione, infrastrutture. Spesso in questi scenari, l'inerzia urbanizzatrice dovuta a tali dinamiche tende a generare spazi con alti livelli di densità edificatoria, superando la capacità di risposta di infrastrutture e risorse naturali locali. 
$\mathrm{Ne}$ è un chiaro esempio la situazione presente lungo la fascia costiera mediterranea della Spagna (Figura 2).

Figura 2. Densità abitativa nelle comunità autonome della costa mediterranea spagnola.

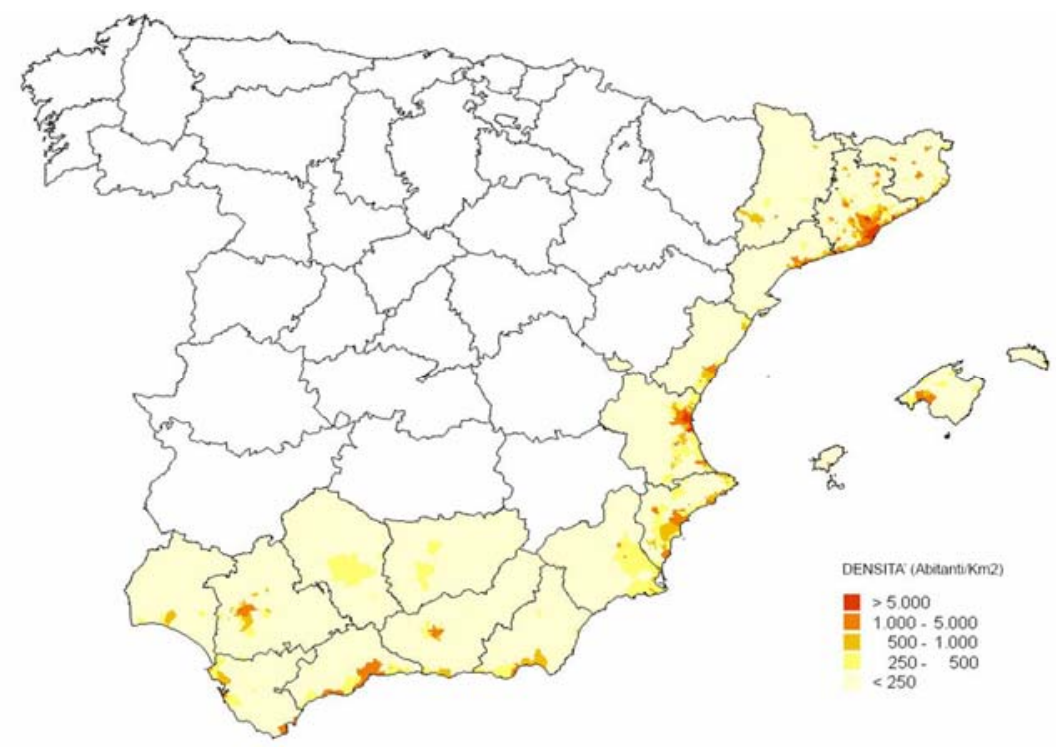

Fonte: workshop: El Proceso de Urbanización en la Costa Mediterránea Española.

È evidente che la vicinanza al mare e la disponibilità di aree balneari, quali attrattori turistici, agisce da "fattore modellante" sulla forma urbana, dato che un territorio, per la sua natura dinamica, tende a trasformarsi ed adattarsi alle esigenze contingenti e spaziali. II cambiamento e la crescita della domanda di territorio costiero, nello scenario post-moderno, è uno dei principali problemi di connessione tra attività di pianificazione e sostenibilità ambientale di zone costiere; industria, edificazione, trasporto, turismo, possono cambiare l'equilibrio delle interazioni terra-mare.

II recente fenomeno di speculazione edilizia in atto lungo la costa mediterranea spagnola ${ }^{4}$, mostra chiaramente come anche determinate condizioni geografiche incidano su fenomeni di crescita, nonché sull'aspetto e sulla definizione di nuove "forme" di città e territori. La sovrapposizione di diverse strutture, la complementarità tra elementi puntuali, lineari e di connessione della struttura urbana, la sintassi spaziale e la geografia, unite a dinamiche speculative, generano nuovi modelli di urbanizzazione. Esistono però delle invarianti storicogeografiche e strutturali che nella loro composizione determinano una tendenza morfologica insediativa e sarebbe pertanto possibile individuare alcuni "modelli" di città contemporanea, funzionale o formale, riconoscibili e ripetibili nel territorio.

Lo studio della morfologia urbana potrebbe rappresentare un utile strumento di conoscenza delle attuali manifestazioni urbanistiche, delle tendenze di crescita e delle varie situazioni presenti nelle realtà territoriali contemporanee.

\footnotetext{
${ }^{4}$ Sono state realizzate o iniziate circa tre milioni di case in Spagna negli ultimi quattro anni, di cui 812.000 nel 2005, e circa la metà di queste si trovano lungo la costa. Secondo alcune stime, nel paese avviene il $40 \%$ di tutta l'attività costruttiva europea.

II boom è alimentato in parte dalla domanda degli europei del nord per case in cui ritirarsi da pensionati sul Mediterraneo. Attirati dal clima mite, dalle abitazioni relativamente economiche, dalla facilità di aggirare le tasse conducendo le operazioni in nero, gli stranieri in alcuni piccoli centri costituiscono fino al $70 \%$ della popolazione.
} 


\section{Obiettivo}

II presente articolo propone essenzialmente una riflessione sulla forma e sui rapporti che legano tra loro città e territori urbanizzati nello scenario del moderno sviluppo dei centri abitati, per definire la forma dei nuovi insediamenti e dei nuovi confini urbani, che coincidano con la città o che siano di dimensioni differenti, più piccoli o più grandi dell'ambito municipale, quartieri o aree metropolitane ad esempio, ed associare ad una forma urbana un modello numerico derivato da analisi statistiche, per riflettere, infine, su fenomeni di diffusione ${ }^{5}$, dispersione ${ }^{6}$, frammentazione o concentrazione, in atto nell'attuale scenario urbano, attraverso il confronto di modelli.

Uno studio morfologico ${ }^{7}$ dunque, che presuppone un'astrazione, a partire dall'immagine esterna di un poligono urbano e dal suo aspetto visibile che si caratterizza come invariante, per identificare la forma attraverso la struttura e ricercarne i caratteri di immutabilità ai vari livelli di scala, fornendo perciò una concettualizzazione di un modello in cui la forma viene intesa come composizione di parti ed emerge dalle connessioni fra i suoi elementi e attraverso relazioni di somiglianza, di prossimità, di simmetria, di chiusura, di continuità ecc.

L'analisi si riferisce all'insieme di elementi che compongono le parti di un "disegno urbano", considerando tutte le parti costitutive di un insediamento quali: infrastrutture, aree edificate omogenee, vuoti, pieni e le relazioni di interconnessione tra essi, storiche, funzionali e morfologiche.

Si intende stabilire un criterio di lettura dei fenomeni di sviluppo in atto lungo la fascia costiera della Regione Metropolitana di Barcellona (RMB) con la "formalizzazione" di schemi interpretativi dei diversi insediamenti presenti su di un territorio antropizzato specifico, concentrando l'attenzione sui processi di edificazione recenti e tuttora in corso che determinano la formazione di specifiche caratteristiche tipologiche e sul tipo di tendenza dello sviluppo delle città e degli ambiti territoriali presenti in tale scenario.

\section{Metodologia di analisi}

Molti studi si propongono di analizzare i tessuti insediativi sulla base di opportuni indicatori quantitativi per supplire alle manchevolezze dovute alla complessità dei fenomeni da analizzare e alla molteplicità di contesti territoriali esistenti. ${ }^{8}$ La forza morfogenetica di tutte le categorie di tessuto urbano e le relazioni tra le parti urbane omogenee e gli elementi strutturali, indicano le prospettive più importanti per la comprensione delle regole formali riconoscibili all'interno dei margini di un'area artificializzata. Ciò però richiede una serie di distinzioni, considerazioni ed ipotesi di lettura alquanto complesse e sfaccettate, dovute alla variabilità delle situazioni e alle dinamiche esistenti.

\footnotetext{
${ }^{5}$ Con il termine diffusione si indica in genere la crescita successiva di piccoli e medi centri urbani collocati negli anelli periferici delle aree metropolitane, oppure in zone lontane dai centri principali, facendo prevalere ad un modello urbano compatto e centralizzato un modello privo di gerarchie formali facilmente identificabili.

${ }^{6}$ Possiamo definire invece la dispersione come un fenomeno di frammentazione esasperata, che "attiene alla casualità delle nuove localizzazioni, alla frammentazione della forma urbana, al bricolage della pianificazione urbanistica e territoriale avulso da ogni principio di economia delle risorse territoriali, alla trama del territorio" (Mauro Baioni).

${ }^{7}$ Per quanto concerne gli studi morfologici urbani, ad esempio, la geometria urbana tradizionale si avvale di interfacce frattali (Batty e Longley, 1994; Bovill, 1996; Frankhauser, 1994).

${ }^{8}$ La variabilità dei fenomeni urbani spesso si avvale di indici descrittivi statistici sintetici per confrontare i vari profili descrittivi di aree differenti; tali indici sono argomento di studio in numerosi filoni dell'analisi territoriale. In questa direzione si collocano il filone degli 'indicatori' (Bertuglia, Clarke e Wilson, eds., 1994, Buckley e Mookherjee, 1996, Sawiki e Flynn, 1996), nell'ambito del quale particolare attenzione è rivolta alla definizione di 'profili descrittivi delle situazioni locali', e le recenti applicazioni nel campo dell'analisi spaziale (Anselin, 1995, Fotheringham, Charlton e Brundsdon, 1996), volte ad approfondire il contributo specifico 'dei diversi elementi locali' all'andamento e/o alle caratteristiche complessive di un fenomeno.
} 
Nello studio qui presentato ci si riferirà sostanzialmente a due grandi classi di analisi: una di tipo quantitativo, che si avvale di indici descrittivi ed analisi statistiche ed una di tipo qualitativo basata sulla rappresentazione di schemi di analisi grafica volti a descrivere i valori numerici previamente calcolati. Saranno presi in esame indici di complessità formale e frammentazione, percentuale di suolo urbanizzata e componente demografica, applicati ai municipi della RMB, valutandone l'andamento al passare da zone più strettamente connesse al mare a quelle più interne. Divideremo l'analisi quantitativa in tre passaggi dettagliati: una di tipo quantitativo propriamente detta, una di tipo "morfometrico" ed una analisi strutturale, per valutare le relazioni numeriche tra gli indici suddetti; mentre l'analisi qualitativa esaminerà l'aspetto estetico dei tessuti "artificializzati" delimitati da poligoni di urbanizzazione.

Saranno oggetto di analisi le "forme", urbane e territoriali, presenti lungo la fascia costiera mediterranea catalana, che fanno parte della Regione Metropolitana di Barcellona (Figura 3).

\section{Figura 3. Cataluña e Regione Metropolitana di Barcellona (RMB).}

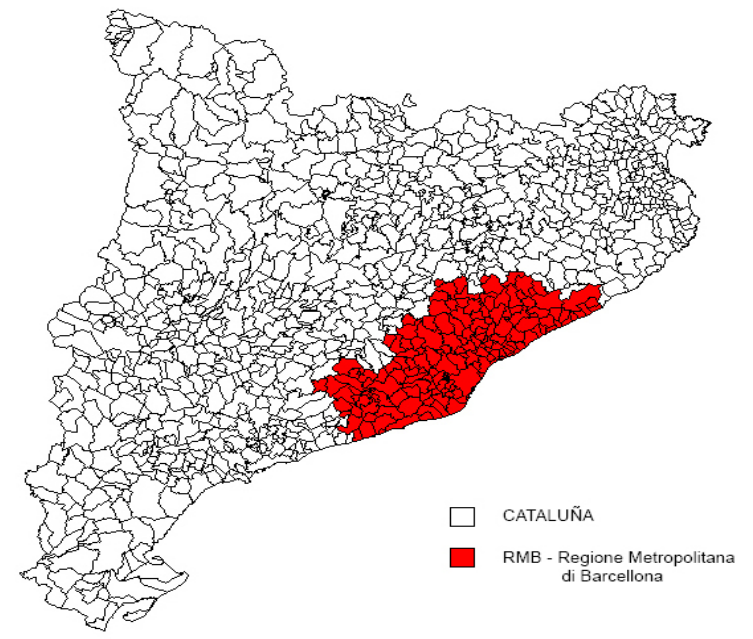

Fonte: Elaborazione propria

L'analisi conoscitiva si articolerà inoltre su due livelli distinti ma fortemente complementari relativi, in particolare, a due scale di definizione di ambito: la prima riferita alla scala urbana vera e propria, considerando la città in sé e con riferimento alla divisione amministrativa municipale; la seconda rivolta ad un ambito sovra-municipale, territoriale quindi, dove le relazioni tra i diversi nuclei urbani danno vita a differenti tipi di modelli formali e funzionali.

Per quanto riguarda tale ambito sovra-municipale si prenderà in considerazione il rapporto funzionale tra più municipi calcolato in base ad un Valore di Interazione (V.I.) tra essi, in grado di generare l'unione di più aree urbane contigue, in una entità unica di dimensioni maggiori che prenderà il nome di protosistema.

\subsection{Ambito sovra-municipale funzionale: protosistema.}

Quella di considerare il protosistema come campo di studio a livello sovra-municipale, in base ad interazioni funzionali territoriali, è una ipotesi tra le varie possibili, così come ad esempio si sarebbe potuto considerare l'area metropolitana, o le divisioni provinciali ${ }^{9}$.

\footnotetext{
${ }^{9}$ II protosistema, riferendosi a valori di interazione funzionale tra organismi urbani contigui, genera strutture territoriali fortemente relazionate tra loro, con caratteristiche interne simili e dimensionalmente più piccole rispetto ad ambiti amministrativi, in cui le connessioni tra municipi tendono ad essere meno consistenti e spesso arbitrarie.

II Valore di Interazione, simmetrico, tra due municipi i e j, pondera i flussi lavorativi tra municipi stessi in base alle rispettive masse, perciò le relazioni locali sono più forti e "predominano" sulle gerarchie amministrative.
} 
La metodologia usata per la generazione dei protosistemi ${ }^{10}$ considera un Valore di Interazione tra due municipi "i" e "j", in relazione tra loro, con riferimento ai flussi migratori lavorativi giornalieri. Le unità che formano un protosistema (Figura 4) vengono raggruppate secondo il più alto Valore di Interazione (V.I.) tra due municipi "i" e "j" seguendo un processo iterativo di calcolo, e imponendo la condizione di contiguità tra municipi ${ }^{11}$ :

$$
\text { V.I. } i j=\frac{F_{i j}^{2}}{P O R_{i} \cdot L T L_{j}}+\frac{F_{j i}^{2}}{P O R_{j} \cdot L T L_{i}}
$$

F: Flusso (spostamenti lavorativi)

POR: Popolazione Occupata Residente (in spagnolo: Popolación Ocupada Residente).

LTL: Centri di lavoro Localizzati (in spagnolo: Lugares de Trabajo Localizado).

La finalità è quella di generare ambiti sovra-municipali che presentano, tra loro, le relazioni più forti di interazione funzionale.

Figura 4. Protosistemi nella Regione Metropolitana di Barcellona (RMB).

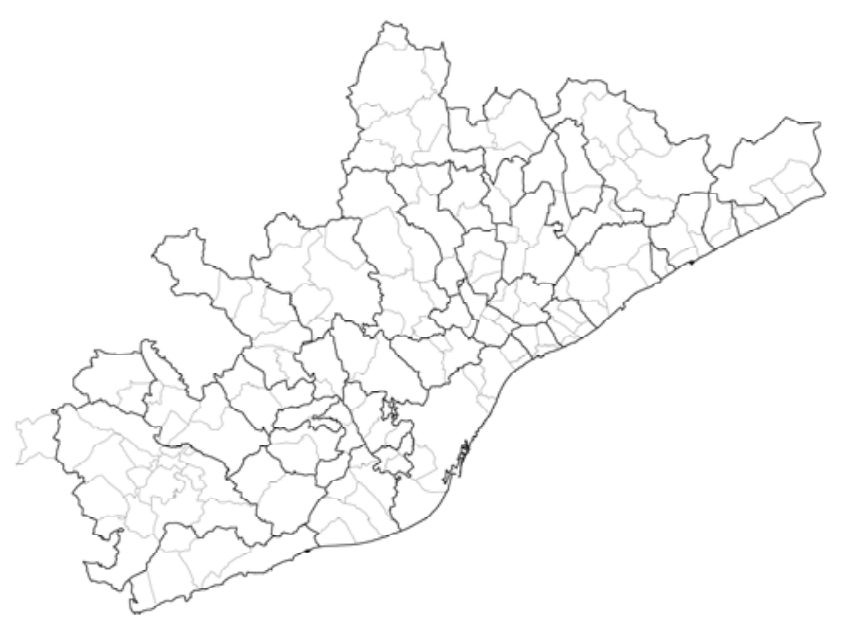

Fonte: Cap a una nova organització territorial de catalunya. Cladera, Josep Roca; Bergadà, Montserrat Moix.

\subsection{Dati quantitativi utilizzati per l'analisi.}

I dati usati per l'analisi sono quelli rilevati attraverso il progetto CORINE Land Cover 2000, un progetto di iniziativa congiunta dell'Agenzia Europea dell'Ambiente e della Commissione Europea che ha interessato 26 paesi e che prevedeva l'acquisizione della copertura del suolo, a livello europeo, attraverso immagini satellitari Landsat 7ETM+, la fotointerpretazione delle stesse e la classificazione del suolo.

CORINE 2000 fornisce una classificazione del suolo divisa in 5 livelli:

10 Protosistemi continui delimitati, in Spagna, applicando questa metodologia: CAT(Cataluña):195 protosistemi; VAL(Valencia): 122 protosistemi; BAL(Baleari): 15 protosistemi; MUR(Murcia): 14 protosistemi; AND(Andalucia): 185 protosistemi.

11 Per ogni municipio si cerca un altro municipio col quale risulti avere il valore di interazione più alto, ed in maniera iterativa si generano "catene" di municipi, che si chiudono quando un municipio presenta la massima interazione funzionale con un altro che già appartiene ad un gruppo, dando vita cpsì ai protosistemi.

Le dimensioni delle "catene" uniscono come minimo due municipi, non essendo perciò possibile l'esistenza di un protosistema formato da un solo municipio. 


\section{SUPERFICI ARTIFICIALI}

1.1. Zone urbanizzate di tipo residenziale: Zone residenziali a tessuto continuo; Zone residenziali a tessuto discontinuo e rado.

1.2. Zone industriali, commerciali ed infrastrutturali: Aree industriali, commerciali e dei servizi pubblici e privati; Reti stradali, ferroviarie e infrastrutture tecniche ${ }^{12}$; Aree portuali; Aeroporti.

1.3. Zone estrattive, cantieri, discariche e terreni artefatti e abbandonati: Aree estrattive ${ }^{7}$; Discariche ${ }^{7}$; Cantieri.

1.4. Zone verdi artificiali non agricole: Aree verdi urbane; Aree ricreative e sportive.

\section{SUPERFICI AGRICOLE}

3. TERRITORI BOSCATI E AMBIENTI SEMI-NATURALI

4. ZONE UMIDE

5. CORPI IDRICI

Attraverso CORINE 2000 otteniamo poligoni di urbanizzazione (Figura 5) in formato vettoriale, digitalizzando le aree artificializzate mediante processi di remote-sensing.

È necessario evidenziare che sono stati utilizzati poligoni "omogeneizzati" che comprendono, cioè, tutte le superfici artificializzate senza distinguere, all'interno di esse, le varie sub-zone e che ciò può rappresentare un limite all'analisi, la quale pero mira soprattutto a mettere in campo una metodologia di studio perfettibile e auspicabilmente soggetta ad approfondimenti futuri ${ }^{13}$.

Figura 5. Poligoni di urbanizzazione nella RMB.

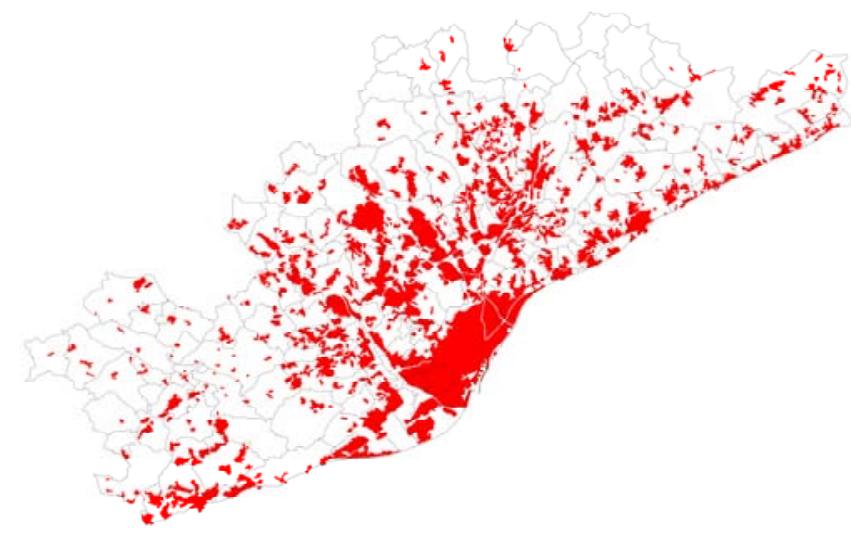

Fonte: Progetto CORINE Land Cover 2000.

\subsection{Analisi quantitativa}

\subsubsection{Analisi quantitativa}

Per l'analisi quantitativa propriamente detta, sono stati utilizzati un indice di occupazione del suolo, considerando il rapporto tra superficie artificializzata e superficie di pertinenza della stessa (a seconda che si consideri l'area municipale o quella protosistemica) e la densità abitativa $^{14}\left(\mathrm{ab} . / \mathrm{m}^{2}\right)$ calcolata come rapporto tra la popolazione residente e la superficie urbanizzata considerata.

\footnotetext{
${ }_{12}^{12}$ Non inclusi nel suolo urbanizzato.

13 Tra le classi non incluse nel calcolo, sono presenti reti ferroviarie e grandi arterie stradali, che in uno studio successivo potrebbero essere interessante oggetto di una analisi particolareggiata, al fine di stabilire caratteristiche e relazioni esistenti tra la "colonna vertebrale" degli organismi territoriali analizzati e le parti che li compongono.

${ }^{14} \mathrm{~L}$ 'indice di densità calcolato è un indice statico e netto, riferito cioè solo alla popolazione residente e calcolato non su l'intera area municipale ma sulla superficie urbanizzata.
} 
Occupazione di Suolo

$O S=\frac{S U\left(m^{2}\right)}{S\left(m^{2}\right)}$

Densità abitativa (netta)

$D=\frac{\text { Pop. }(n)}{S U\left(m^{2}\right)}$

\subsubsection{Analisi strutturale}

L'analisi strutturale si basa sul concetto di entropia ${ }^{15}$ che fornisce un indice di Frammentazione della struttura urbana a seconda del numero di poligoni che la compongono e del "peso" che ogni poligono ricopre in un determinato scenario. L'indice entropico rientra nella categoria di tutta una serie di indicatori descrittivi statistici che misurano la variabilità di specifiche condizioni quali: eterogeneità, dispersione, equilibrio e concentrazione. L'uso di tali indici serve ad ottenere misure descrittive sintetiche, delle distribuzioni spaziali di un fenomeno in un'area, finalizzate a consentire il confronto tra spazi diversi.

L'indice di entropia $\mathrm{H}$ ci permette di misurare la probabilità che un poligono di urbanizzazione sia presente o meno su un dato territorio, ossia il numero ed il peso dei soggetti che compongono un dato sistema. Più alta sarà la probabilità, maggiore sarà il grado di frammentazione urbana, in base anche alla grandezza dei singoli nuclei che stabiliscono quindi un ordine di importanza tra essi.

Se il valore di $\mathrm{H}$ è uguale $\mathrm{a} 0$, cioè la probabilità $\mathrm{P}=1$, siamo in presenza di un solo poligono di urbanizzazione e quindi "zero" frammentazione (considerando non la forma ovviamente ma solo il numero dei poligoni presenti e la superficie occupata).

La formula usata per l'entropia è quella proposta da Shannon ${ }^{16}$ :

Entropia

$H=-1 \sum_{i=1}^{n}\left[P_{i}\left(\ln P_{i}\right)\right]$

P: probabilità (rapporto tra la superficie di un poligono di urbanizzazione e la somma della superficie totale di tutti i poligoni)

$\mathrm{n}=$ numero di poligoni.

\footnotetext{
${ }^{15}$ L'entropia è un concetto della fisica che misura il grado di disordine. Una scatola dei fiammiferi sparsi a caso sul pavimento fornisce un modello con una alta entropia visiva. L'entropia si ridurrà nel momento in cui i fiammiferi saranno allineati in una configurazione più ordinata. Non deve essere un modello rettangolare, ma potrebbe assomigliare ad una ragnatela o ad una spirale. Le simmetria matematiche - in questo caso translazionali, di rotazione, radiali, o a spirale generano un ordine alla grande scala, ordine che abbassa l'entropia visiva.

${ }_{16}$ L'indice entropico $(H)$ introdotto da Shannon nel 1948 è una misura di eterogeneità su scala logaritmica; essa assume valore minimo zero quando tutte le unità del collettivo presentano la stessa modalità e valore massimo $(\log n)$ nel caso di equiripartizione delle unità tra le diverse modalità. (Eugenio Novelli, Sylvie Occelli)
} 


\subsubsection{Analisi morfometrica}

Gli indici di forma sono quelli che possono descrivere la morfografia di un poligono di urbanizzazione attraverso $\mathrm{i}$ rapporti tra le sue grandezze geometriche elementari: area e perimetro; si possono ottenere in questa maniera parametri di confronto tra poligoni di forma e dimensioni differenti. Tra questi indici i più noti ed internazionalmente riconosciuti sono l'indice di Gravelius, l'indice di Miller (Linsley, 1988) ed il rettangolo equivalente (Roche, 1963).

Useremo l'indice di Gravelius ${ }^{17}$, calcolato in base al rapporto tra il perimetro del poligono considerato e la circonferenza del cerchio di pari area, per determinare il grado di complessità formale delle singole unità componenti il complesso in esame; "aggiusteremo" però la formula introducendo una ponderazione della stessa, che misuri l'importanza di ogni nucleo all'interno del sistema di modo che, in definitiva, il risultato ottenuto descriva il grado di complessità formale dell'intero organismo urbano in base alle sue gerarchie intrinseche.

L'indice di complessità formale (Kf d'ora in avanti) può risultare un utile strumento di calcolo per descrive il grado di compattezza di una urbanizzazione nel suo complesso, nonché la sua tendenza formale; quanto più la forma del poligono sarà compatta o poco "regolare", cioè quanto più piccolo sarà il suo perimetro rispetto alla superficie, tanto più i valori dell'indicatore tenderanno all'unità. Il caso limite è quello di poligoni di forma perfettamente circolare con $K f=1$; quanto più il contorno dei poligoni di urbanizzazione sarà frastagliato ed irregolare, tanto più il valore di $\mathrm{Kf}$ si allontanerà dall'unità crescendo $(\mathrm{Kf} \geq 1)$.

Indice di complessità di forma

$K f=\frac{\sum_{i=1}^{n}\left[\frac{P_{i}}{2 \sqrt{ }\left(\pi A_{i}\right)} * A_{i}\right]}{\sum_{i=1}^{n} A_{i}}$

$P=$ Perimetro del poligono di urbanizzazione

$A=$ Area del poligono di urbanizzazione

$\mathrm{n}=$ numero di poligoni di urbanizzazione

$f=$ Forma

\subsection{Analisi qualitativa}

L'analisi qualitativa fa riferimento ad un campo sicuramente più teorico-concettuale, per cui le considerazioni fatte analizzando modelli urbani e territoriali, in merito a tipo di struttura, caratteristiche morfologiche, rapporti di interconnessione, ecc., si avvarranno di un background conoscitivo personale, per individuare determinate caratteristiche invarianti dei sistemi.

Ciò potrebbe risultare, in parte, influenzato da una inevitabile componente soggettiva, per cui si farà ricorso ad un supporto scientifico di analisi statistiche per l'elaborazione dei dati numerici derivati dall'analisi quantitativa, che giustificherà la classificazione di gruppi coerenti di strutture urbane, definite a partire da schemi formali. Dal punto di vista metodologico infatti, anche il solo uso di indicatori descrittivi privo di una lettura critica, qualitativa, potrebbe essere non significativo nonché riduttivo e la loro capacità esplicativa dimostrarsi insufficiente per spiegare fenomeni di tale complessità.

\footnotetext{
${ }^{17}$ Gravelius $=P / 2 \sqrt{ }(\pi \mathrm{A})$, dove $P$ è il perimetro di un poligono ed $A$ è la sua superficie.
} 
Lo scopo è di riconoscere la consistenza del territorio urbanizzato in termini di pieni e di vuoti, ma soprattutto in termini di forma, con l'intento di definire e graficizzare una serie di tracciati morfologicamente riconoscibili pur in diversi contesti geografici. La forma ha la caratteristica infatti, di manifestarsi attraverso una sequenza di discontinuità qualitative nello spazio in cui vive ed il complesso di tali discontinuità "disegna" la struttura di un sistema e del rispettivo processo dinamico che lo ha generato, marcando il confine tra margini naturali o artificiali.

In pratica il lavoro consisterà in:

- ipotizzare l'esistenza di modelli urbani con caratteristiche tipologiche comuni, secondo aspetti specifici;

- calcolare i valori di complessità formale, entropia, occupazione di suolo e densità a livello municipale o territoriale, mediante l'uso di tecnologie GIS;

- confrontare tra loro i risultati mediante grafici di dispersione ed istogrammi, e rappresentare con l'indice di Pearson la correlazione che intercorre tra le variabili considerate, sapendo che quanto più bassa è tale relazione tanta più complementarità esiste tra gli indici;

- mappificare i valori riscontrati, in modo da ottenere un quadro visivo delle distribuzioni spaziali dei singoli indici nella RMB;

- classificare gruppi simili, in base alle caratteristiche considerate, mediante un'analisi cluster; in cui introdurremo i coefficienti Kf, H, OS, D;

- confrontare i clusters calcolati, con i modelli teorici previamente ipotizzati, attraverso analisi di caso.

Aspetto interessante dell'analisi sarà infine la valutazione del comportamento degli organismi urbani al cambio di scala, passando dall'ambito municipale a quello protosistemico.

\section{Modelli di urbanizzazione: scala urbana e scala territoriale.}

È possibile distinguere le varie zone che organizzano un nucleo urbano, in base al tipo di tessuto, in cui i singoli elementi costitutivi sono strettamente relazionati tra loro e occupano posizioni reciprocamente dipendenti formando, nell'insieme, una maglia tendenzialmente continua e interconnessa e conferendo ad un impianto peculiarità "riassumibili" in forme che potremmo classificare per linee generali: reticolare, radiale, organico o complesso, semplice, composto, lineare, poli-nucleare, tentacolare o a ventaglio.

Sempre più di frequente, in epoca contemporanea, le parti componenti delle città seguono proprie tendenze formali in maniera autonoma, dando vita a configurazioni frammentate in cui le urbanizzazioni si realizzano per giustapposizione casuale di parti, senza un preciso ed identificabile disegno di base, "casualmente" e senza una logica pianificatoria.

La struttura di un impianto territoriale deriva quindi dal risultato della somma dei singoli nuclei che generano lo spazio, creando degli organismi composti, di tipo compatto o compattato, disperso, diffuso o frammentato, a seconda del grado di continuità con cui si susseguono le agglomerazioni urbane, città o conurbazioni che siano.

Astraendo in forme geometriche l'essenza di organismi urbani, ipotizziamo l'esistenza di alcuni modelli di urbanizzazione, classificandoli in gruppi con caratteristiche comuni di compattezza, frammentazione, dimensione e relazione fra le parti.

- A livello municipale (scala urbana), possiamo descrivere il comportamento formale degli complessi urbani in tre grandi categorie: 

A. Compatto
Poligonale, Circolare, Lineare
B. Compattato in nuclei
C. Frammentato ${ }^{18}$
D. Tentacolare

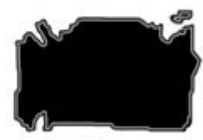

A

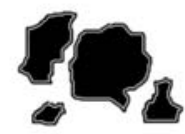

B

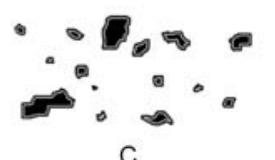

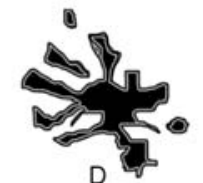

- A livello territoriale (protosistemi), dove le relazioni che più pesano sono di tipo funzionale tra le parti anziché ti tipo formale, possiamo riassumere il comportamento delle strutture in esame in due categorie principali e tre sub-categorie ${ }^{19}$ :

a. Concentrato

b. Disperso

b.1 Disperso Strutturato

b.2 Disperso De-composto

b.3 Disperso Aperto

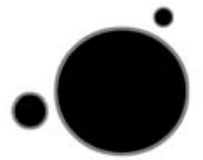

a

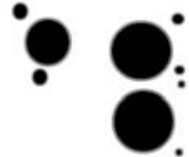

b. 1

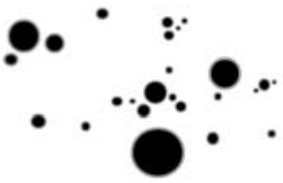

b. 2

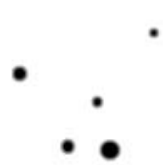

b. 3

\section{Analisi di caso}

\subsection{Municipi}

La prima operazione consiste nel quantificare i valori delle quattro variabili considerate per I'analisi (Kf, H, OS, D) e calcolate sui poligoni di CORINE, attraverso le formule [1], [2], [3], [4], attribuendo tali valori al municipio di appartenenza.

Nel passo successivo, i dati numerici ottenuti, saranno messi in relazione fra loro mediante l'uso di analisi statistiche, considerando di volta in volta le variabili in combinazioni di due per calcolarne la correlazione lineare definita dal coefficiente di Pearson (Tavola 1$)^{20}$.

Per mostrare le distribuzioni spaziali delle variabili, all'interno della RMB, ci avvarremo di un GIS, fissando tre livelli di valori: Alto, Medio, Basso (Figura 6).

\footnotetext{
${ }^{18} \mathrm{Ci}$ troviamo in presenza di modelli di tipo $C$ quando l'ambito considerato è caratterizzato dall'esistenza di grandi superfici di tessuti urbani a morfologia dispersa, tipo urbanizzazioni a blocchi isolati e/o con giardino che difficilmente presentano una relazione strutturante con le reti infrastrutturali e di comunicazione.

${ }_{19}$ Quando si parla di strutture territoriali, che siano "compatte" o disperse, ci si riferisce comunque a sistemi policentrici complessi, poiché formati dalla somma di singoli soggetti, in cui basicamente, al fine di definire un modello, influiscono il grado di dispersione sul territorio ed il grado di frammentazione delle singole parti che costituiscono tale organismo.

${ }^{20}$ Nella matrice di Pearson è possibile osservare che tra occupazione di suolo e densità, la correlazione è alta, come ci si aspetterebbe, ma anche tra occupazione di suolo e complessità formale la relazione risulta sensibile. Ciò potrebbe essere spiegato dalla mancanza di suolo edificabile che fa si che si utilizzi al massimo lo spazio "interstiziale", residuale, tra gli spazi non edificabili; ma la complessità formale di una città probabilmente dipende anche dal maggior numero di parti che la compongono, laddove è evidente che sistemi più grandi presentano maggior varietà di tessuti urbani che nel giustapporsi generano, specie in anelli periferici, un contorno irregolare senza necessariamente generare dispersione.
} 
Tavola 1. Matrice di correlazione di Pearson.

\begin{tabular}{|c|c|c|c|c|c|}
\hline & & Coeff_Forma & Entropia & Occ_Suolo & $\begin{array}{l}\text { Densità_- } \\
\text { Urbano }\end{array}$ \\
\hline \multirow[t]{3}{*}{ Coeff_Forma } & Pearson Correlation & 1 &, 069 &, $351^{* \star}$ & ,100 \\
\hline & Sig. (2-tailed) & & ,384 & ,000 & 202 \\
\hline & $\mathrm{N}$ & 163 & 163 & 163 & 163 \\
\hline \multirow[t]{3}{*}{ Entropia } & Pearson Correlation & ,069 & 1 &,- 149 &,$- 269^{* *}$ \\
\hline & Sig. (2-tailed) & ,384 & & 057 &, 001 \\
\hline & $\mathrm{N}$ & 163 & 163 & 163 & 163 \\
\hline \multirow[t]{3}{*}{ Occ_Suolo } & Pearson Correlation & $351^{* *}$ &,- 149 & 1 &, $637^{\star *}$ \\
\hline & Sig. (2-tailed) &, 000 &, 057 & &, 000 \\
\hline & $\mathrm{N}$ & 163 & 163 & 163 & 163 \\
\hline \multirow[t]{3}{*}{ Densità_Urbano } & Pearson Correlation & ,100 &,$- 269^{* *}$ &, $637^{* *}$ & 1 \\
\hline & Sig. (2-tailed) & ,202 &, 001 & ,000 & \\
\hline & $\mathrm{N}$ & 163 & 163 & 163 & 163 \\
\hline
\end{tabular}

${ }^{* *}$. Correlation is significant at the 0.01 level (2-tailed).

Figura 6. Distribuzione spaziale delle variabili.
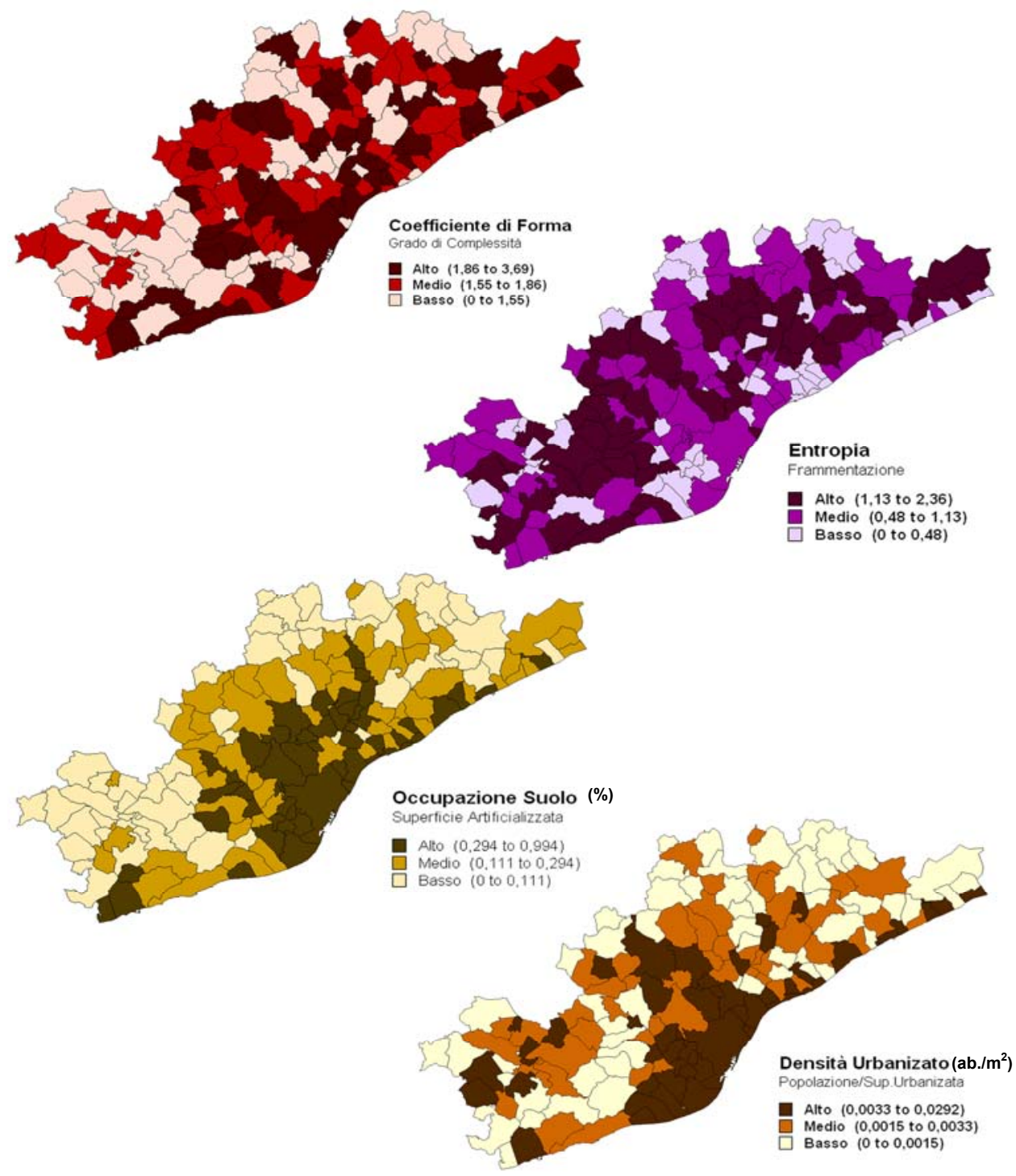

Fonte: Elaborazione propria

MODELLI DI URBANIZZAZIONE COSTIERA: MORFOLOGIA E COMPLESSITÀ STRUTTURALE, A SCALA 
Se partiamo dall'idea che i processi di urbanizzazione attualmente in atto lungo le aree costiere, seguono meccanismi di evoluzione diversi da modelli di urbanizzazione dell'entroterra, a causa di differenti meccanismi costituenti, sempre più preponderanti e che il contatto diretto col mare può influenzare il territorio a seconda della distanza dalla linea di costa, sarebbe possibile dividere la RMB in tracciati paralleli al fronte costiero e valutare l'andamento dei valori delle variabili (Figura 7$)^{21}$.

Figura 7. La RMB in quattro linee di costa.

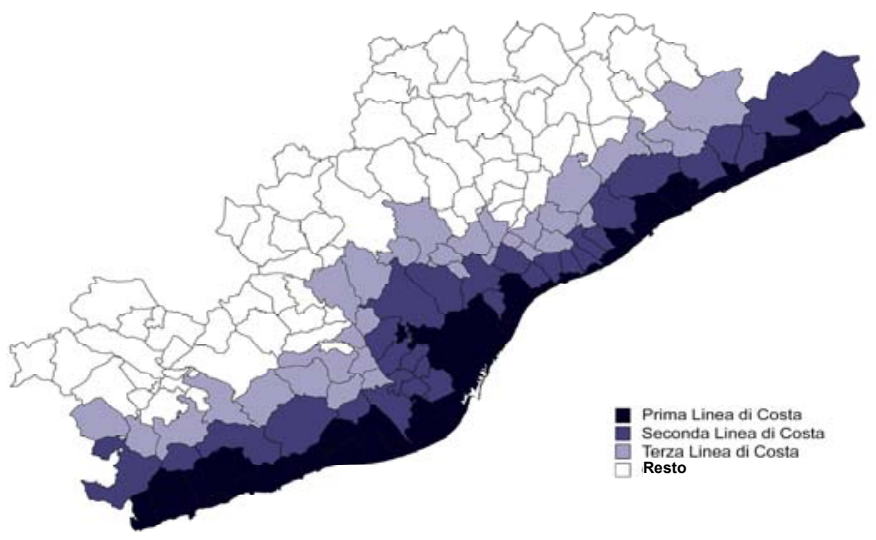

Fonte: Elaborazione propria

Il diagramma a barre, riferito alle variabili di forma, entropia, occupazione di suolo e densità netta (Tavola 2), mostra la tendenza degli indici lungo tratti paralleli al fronte litorale.

Tavola 2. I valori medi ponderati delle variabili per le quattro linee di costa.

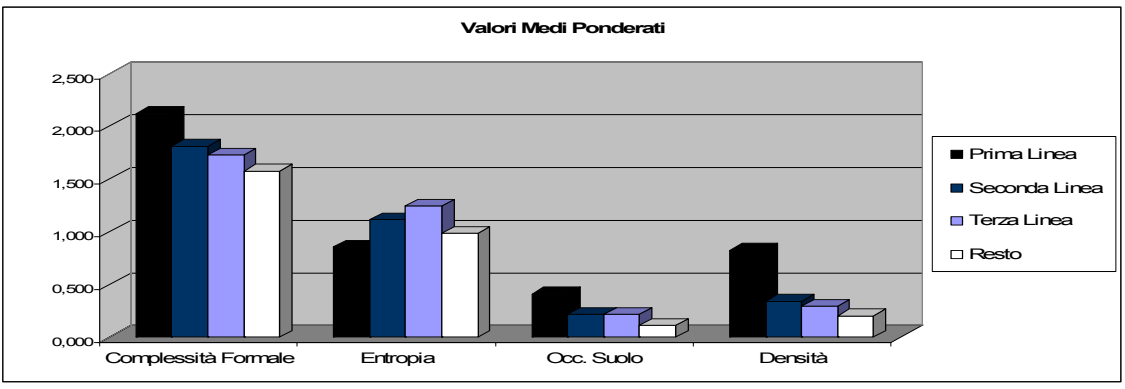

Fonte: Elaborazione propria

Lungo la prima linea pesano soprattutto l'occupazione di suolo, la densità abitativa ed il coefficiente di complessità formale poiché una alta "pressione urbanizzatrice" tende ad "allungare" i poligoni urbani e ad aumentarne perimetro, superficie ${ }^{22}$ ed altezza degli edifici, compattandoli e riducendo il grado di frammentazione ${ }^{23}$, riempiendo gli spazi di interconnessione e sfruttando al massimo la rendita di posizione.

È sempre più evidente, di fatto, la propensione alla formazione di un fronte continuo urbanizzato lungo la fascia litorale.

\footnotetext{
${ }^{21}$ La divisione in quattro fasce è stata pensata in base al "contatto" col mare: diretto per i municipi di prima linea, diretto con i munipici di prima linea, per quelli di seconda e così via. In questo studio è stata trascurata la componente orografica del terreno, che probabilmente genererebbe divisioni territoriali più robuste ma anche considerazioni di altro tipo, che saranno sviluppate in lavori successivi.

${ }^{22}$ La complessità formale è un indice calcolato in base ad una proporzione tra Area e Perimetro di un poligono.

${ }^{23}$ La frammentazione tende ad aumentare con l'aumentare della distanza, ma nell'ultima zona, la più distante, si assiste ad una diminuzione dell'entropia dovuta alla presenza di alcuni importanti municipi quali Terrassa, Granollers o Vilafranca che per effetto della media ponderata dei valori, tendono a "deviare" la tendenza della variabile.
} 
Le variabili considerate per l'osservazione dei modelli urbani, sono state normalizzate e studiate avvalendosi di un'analisi cluster per individuarne i centri e la disposizione, attorno ad essi, di tutti i soggetti coinvolti nell'applicazione.

Si sono realizzate due analisi cluster, la prima di tipo gerarchico, per determinare il numero più opportuno di gruppi da considerare e la seconda di tipo K-means, con cinque classi di raggruppamento (Figura 8 ).

Figura 8. Analisi cluster per municipi.

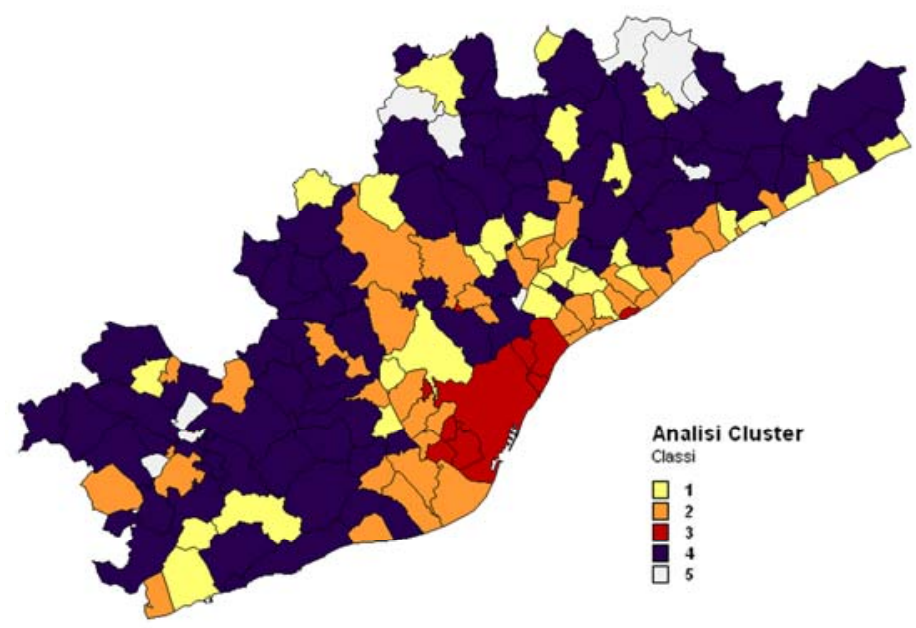

Fonte: Elaborazione propria

Per ognuno dei cinque cluster sono stati estratti i centri delle variabili (Tavola 3) e tracciate le linee di tendenza di ogni gruppo rispetto alle variabili stesse, per poterne confrontare il comportamento per classi omogenee di valori (Tavola 4).

Tavola 3. Centri dei cluster.

\begin{tabular}{ccccc} 
& Coeff_Forma & Entropia & Occ_Suolo & Densità_Urbano \\
\hline Cluster & & & & \\
\hline 1 & 2,34052 & 0,29690 & 0,2590 & 0,002334 \\
2 & 1,66132 & 0,62756 & 0,35873 & 0,006115 \\
3 & 1,77778 & 0,29778 & 0,74544 & 0,018789 \\
4 & 1,64058 & 1,34096 & 0,13242 & 0,001788 \\
5 & 0,31464 & 0,2636 & 0,00573 & 0,000718 \\
\hline
\end{tabular}

Tavola 4. Valori medi delle variabili per i 5 cluster.

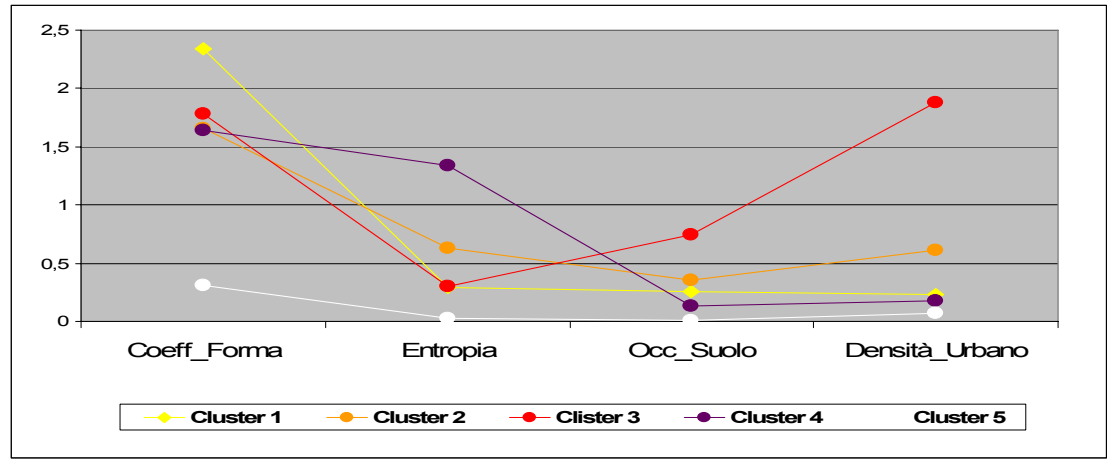

Fonte: Elaborazione propria 
Finalmente compariamo le strutture urbane ipotizzate al capitolo 4: compatto, compattato in nuclei, frammentato e tentacolare, con i gruppi calcolati mediante analisi cluster (Figura 9) assegnando, ad ogni modello, classi di municipi raggruppati in base a caratteristiche similari.

\section{Figura 9. Analisi di caso. Municipi.}

Cluster 3
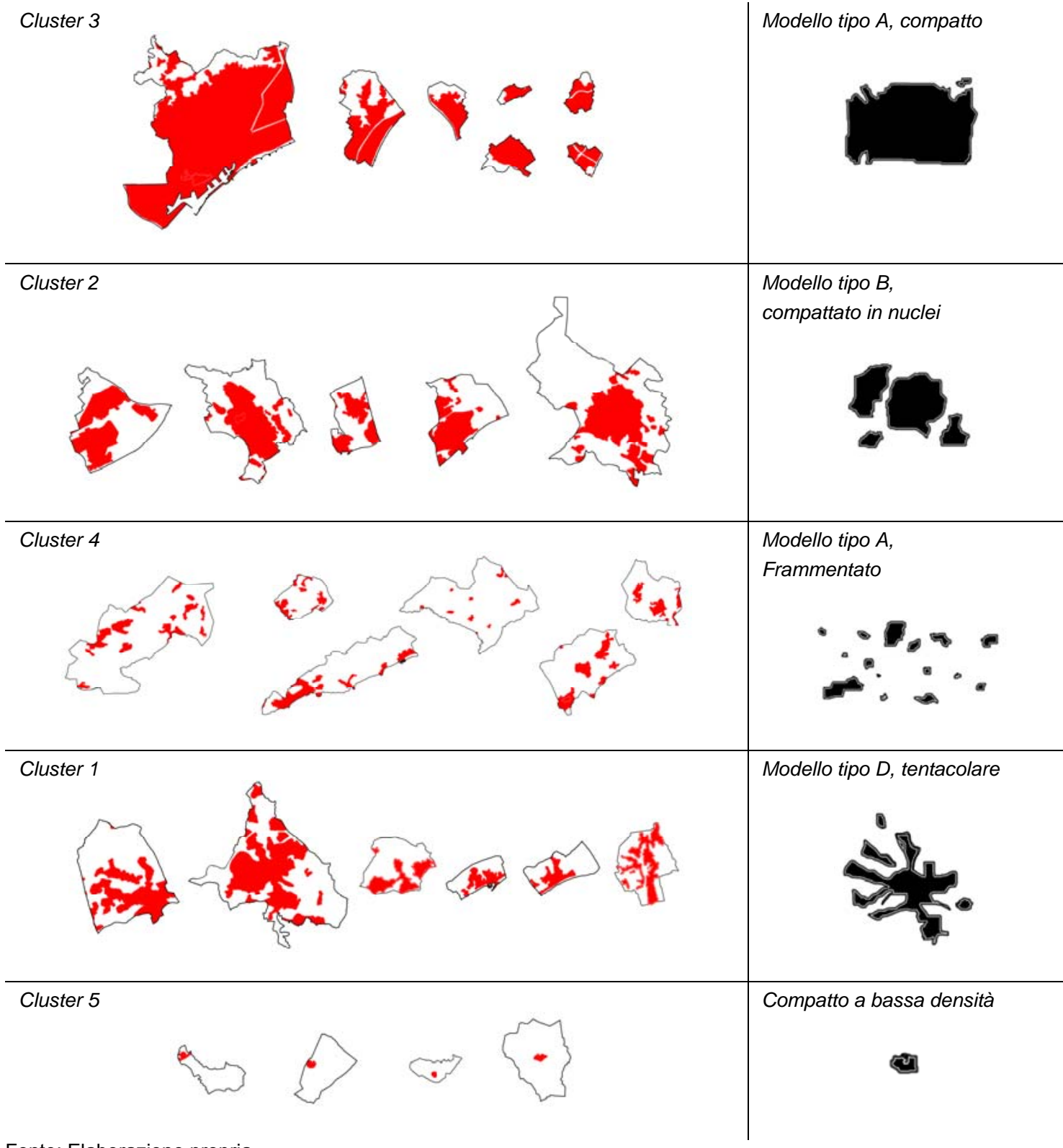

Fonte: Elaborazione propria

Al modello compatto (Tipo A) si riferiscono municipi quali Barcellona con valori $\mathrm{Kf}=2,795$, OS $=$ $0,828, \mathrm{D}=15.400 \mathrm{ab} . / \mathrm{Km}^{2}, \mathrm{H}=0,5 ;$ Badalona con $\mathrm{Kf}=2,381, \mathrm{OS}=0,55, \mathrm{D}=9.980 \mathrm{ab} . / \mathrm{Km}^{2}, \mathrm{H}$ $=0,77$; Santa Coloma de Gramenet con $\mathrm{Kf}=1,980$, OS = 0,6, D = 17.200 ab. $/ \mathrm{Km}^{2}, \mathrm{H}=0,035 \mathrm{e}$ gli altri del cluster 3 , dove la compattezza della struttura è dovuta a livelli alti di occupazione di suolo e densità, mentre la complessità formale elevata dipende dalle corone più esterne del nucleo, in cui le urbanizzazioni di tipo meno intensivo integrate al tessuto consolidato contribuiscono a "sfrangiare" esternamente il profilo generale, ma a mantenere basso il grado di frammentazione (entropia). 
Passando dal modello A a B e quindi a C, si nota come la struttura urbana tenda ad una insistente e successiva rottura, indirizzata verso un policentrismo morfologico, laddove, come in Terrassa, Matarò o Sabadell (Tipo $B$, cluster 2), aumenta il grado di frammentazione $(H=1,21$; $0,9 ; 0,83)$ mentre gli altri valori, pur diminuendo, garantiscono un livello di compattezza del complesso. Nei municipi del cluster 4 (Modello C), invece, abbiamo i livelli massimi di entropia (Tordera $=2,33$; Sant Pere de Ribes $=1,62$; Subiarts $=2,21$ ), causati da un tipo di urbanizzazione a macchia i cui nuclei, in genere, non presentano un rapporto di relazione reciproca con la rete infrastrutturale e dove un polo centrale non si impone come tale e soccombe al dominio di organismi satellite.

II Modello D (cluster 1), al contrario, presenta una sorta di ricucitura del tipo C, dovuta all'esistenza di importanti vie di comunicazione che uniscono i centri del complesso, conferendo al sistema una forma a raggiera (Tentacolare) in cui le urbanizzazioni vengono tenute insieme dalla forza funzionale che "disegna" in qualche modo una morfologia non "satellitare", diminuendo così la frammentazione, aumentando in parte l'occupazione di suolo e la densità, ma soprattutto generando i massimi valori di complessità formale Kf (Vilanova i la Geltrù $=2,85$; Sant Cugat $=2,52$; Palau de Plegamans $=3,07$; Arenys de Mar $=2,85$ ) .

II cluster 5 , poi, in cui tutti i livelli delle variabili risultano bassi, si potrebbe classificare come modello compatto ma di bassa densità, nel quale l'urbanizzazione si concentra in un unico poligono di ridotte dimensioni.

Sui cinque cluster infine, è stata effettuata una analisi Box-Plot (Tavola 5) per verificare la quantità di outliers e quindi il grado di omogeneità dei gruppi stessi, nonché la distribuzione dei soggetti osservati all'interno di ogni insieme.

\section{Tavola 5. Analisi Box-Plot per cluster di variabili.}

Coefficiente di Forma Kf

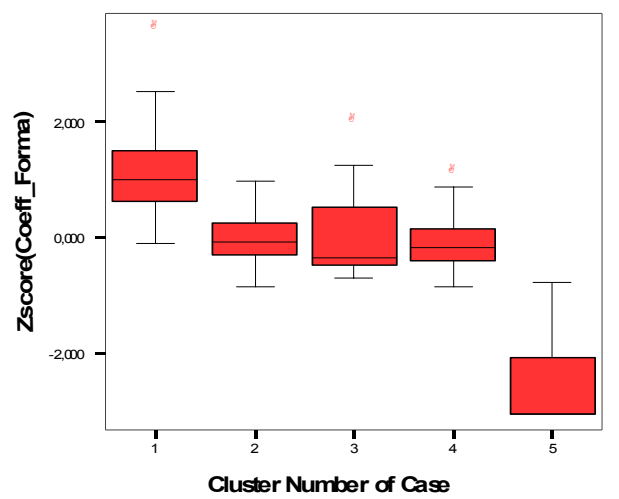

Occupazione Suolo OS

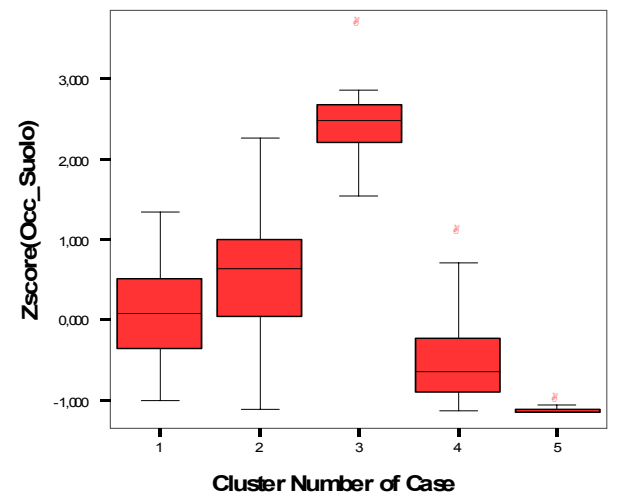

Fonte: Elaborazione propria
Entropia $\mathrm{H}$

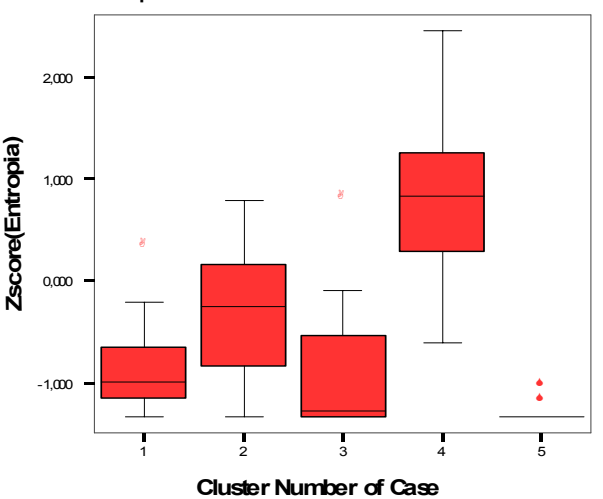

Densità Urbanizzato D

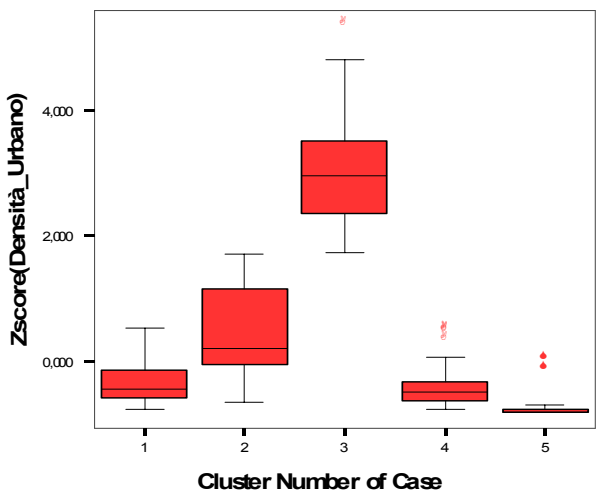




\subsection{Protosistemi}

Così come per i municipi, si è proceduto analizzando la RMB anche a livello sovra-municipale, considerando quale ambito territoriale il protosistema (capitolo 3, paragrafo 3.1) e dopo aver effettuato le varie considerazioni circa le correlazioni tra le variabili ed una analisi di tipo gerarchico, sono stati calcolati sei gruppi omogenei di protosistemi attraverso un'analisi cluster $k$-means (Figura 10).

\section{Figura 10. Analisi cluster per protosistemi.}

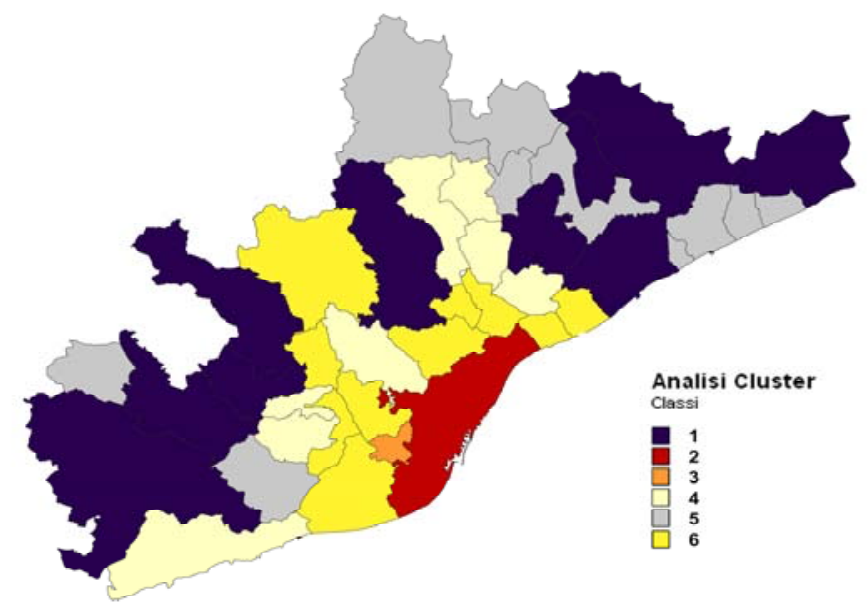

Fonte: Elaborazione propria

Tra i gruppi calcolati si riscontrano certe similitudini tra cluster 2 e cluster 3 e tra il 4 ed il 6 (Tavola 6), con valori alti di occupazione di suolo e densità (maggiori nei gruppi 2 e 3 ) e valori più bassi di entropia, che invece si elevano man mano che ci si allontana dalla costa e da un evidente punto "centrale", rappresentato da Barcellona e dalla sua area territoriale, sottoposto ad una pressione urbanizzatrice elevata.

Dato che questa scala di analisi è generata dal raggruppamento di più soggetti ${ }^{24}$, le strutture che si producono sono sempre di natura polinucleare e spesso la differenza tra i gruppi la fa la presenza o meno di nuclei "dominanti", che tendono a compattare (o meglio a concentrare) il sistema piuttosto che a disperderlo, mediante nuclei equivalenti, in strutture aperte.

Tavola 6. Valori medi delle variabili per i 6 cluster.

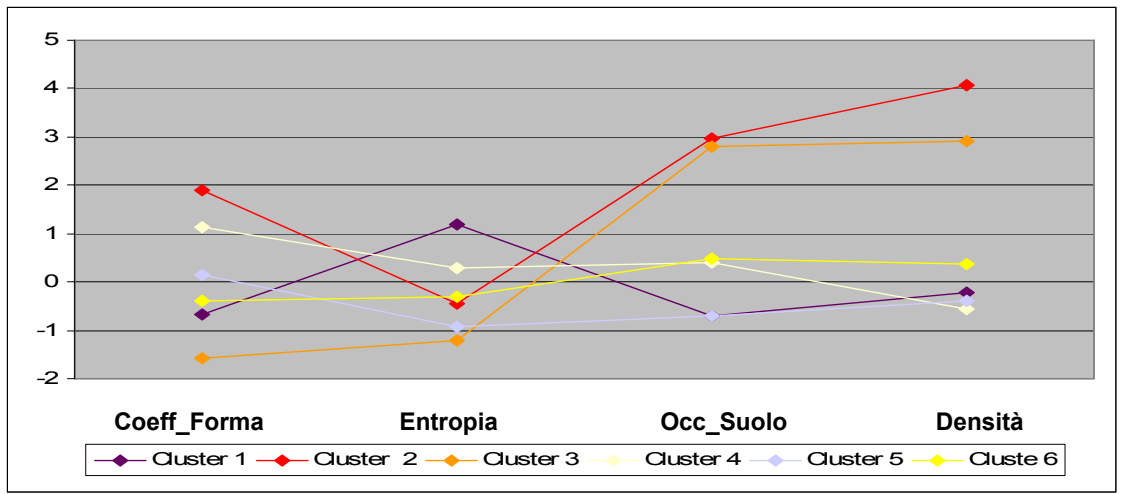

Fonte: Elaborazione propria.

${ }^{24}$ II raggruppamento è stato fatto in base alla somma dei valori di ogni singolo poligono di urbanizzazione, non considerando i nuclei dei protosistemi come entità unitarie quindi ma come somma di singole unità appartenenti ai municipi che compongono gli ambiti territoriali. 
Compariamo le strutture territoriali ipotizzate al capitolo 4: concentrato, disperso strutturato, disperso de-composto, disperso aperto, con i gruppi calcolati nell'analisi cluster (Figura 11).

\section{Figura 11. Analisi di caso. Protosistemi ${ }^{25}$.}

Cluster 2, Cluster 3

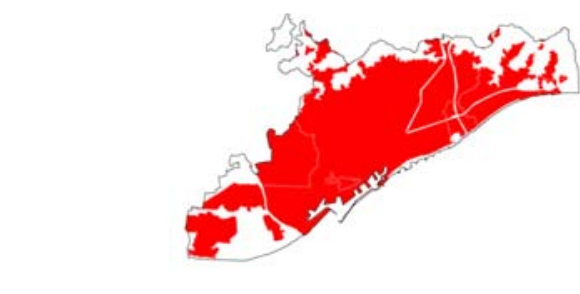

Modello tipo a, Concentrato

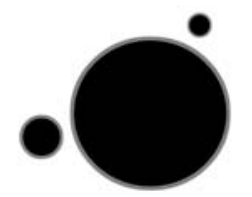

Modello tipo b.1,

Disperso Strutturato

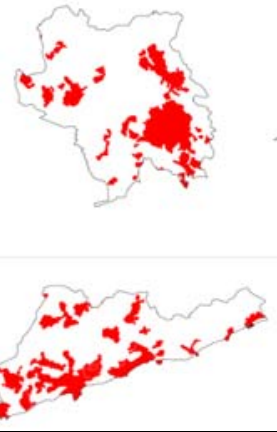

Cluster 1

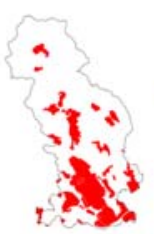

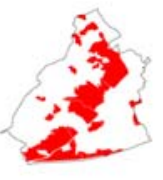

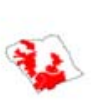

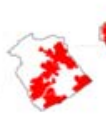
E.
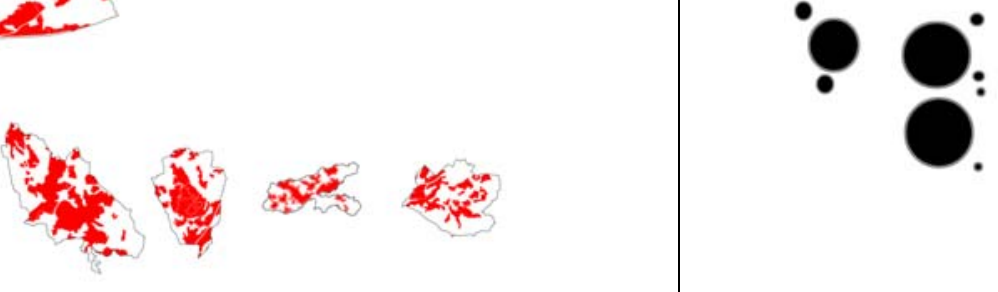

Modello tipo b.2,

Disperso De-composto

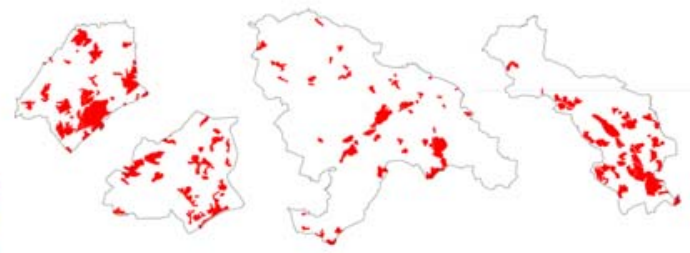

Cluster 5
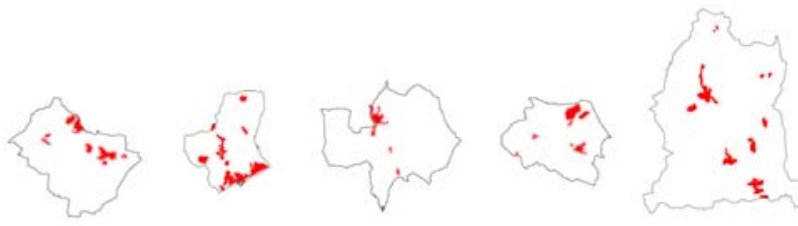

Modello tipo b.3,

Disperso Aperto

Fonte: Elaborazione propria

Se l'analisi cluster municipale ha evidenziato una tendenza comune prevalentemente lungo la fascia costiera e quella territoriale ha posto maggiormente l'accento su Barcellona quale polo di concentrazione di realtà ad alto tasso di occupazione di suolo e densità abitativa, qual è dunque il fattore determinante nella creazione di modelli di urbanizzazione: la componente posizionale, o la presenza di un polo economico...o le due cause insieme? E quale pesa maggiormente?

\footnotetext{
${ }^{25}$ Quella dei protosistemi è una delle ipotesi possibili, proposta per la classificazione di ambiti sovra-municipali, usando un criterio funzionale di unione tra municipi; ma il dibattito interessante attualmente è proprio quello rivolto a definire $\mathrm{i}$ reali confini di interazione che oggi si vanno proporzionando alle dinamiche urbane in continuo divenire, per meglio riflettere sulle cause generatrici di modelli di urbanizzazione in base alla scala di riferimento.
} 


\section{Conclusioni}

II quadro conoscitivo sugli attuali fenomeni di crescita urbana, per essere esauriente, richiederebbe riferimenti a tipi di trasformazioni urbane e territoriali che non dipendono direttamente dai luoghi legati alla residenza o al lavoro. I luoghi di fruizione turistica, ad esempio, si sono estesi enormemente ed $\mathrm{i}$ centri commerciali e ricreativi tendono sempre di più ad occupare la periferia urbana rendendone meno definiti i confini. Spesso abbiamo di fronte fenomeni "degenerativi" che si trascinano già da tempo modificando poco a poco il territorio sul quale viviamo. La città contemporanea, nella quale zone residenziali, commerciali, terziarie e per il tempo libero si giustappongono quasi seguendo un "disordine controllato", è sempre più dispersa sul territorio, aperta in uno spazio senza più centro né periferia ed interrotto da aree residuali e, di frequente, decontestualizzate.

I confini amministrativi si fondono e l'edificazione di una città invade e subisce l'edificazione di realtà limitrofe seguendo una crescita sempre più dinamica ed incontrollata.

Osservando il comportamento dei cluster (Tavola 6; Tavola 8), emerge una chiara tendenza, in zona costiera nella RMB, a subire una pressione urbanizzatrice, dovuta ai suddetti fenomeni di trasformazione, soprattutto lungo due assi: uno parallelo alla linea litorale ed uno perpendicolare ad esso, che coincidono in un centro di pressione rappresentato da Barcellona. L'analisi operata in ambito di protosistemi evidenzia la propensione, a livello territoriale, dell'immediato intorno di Barcellona a concentrarsi in una sorta di piramide con la base collocata sulla costa, dove le percentuali di suolo urbanizzato, la densità abitativa e la complessità formale assumono i valori più alti ${ }^{26}$ (Figura 12).

Figura 12. Pressione di urbanizzazione.

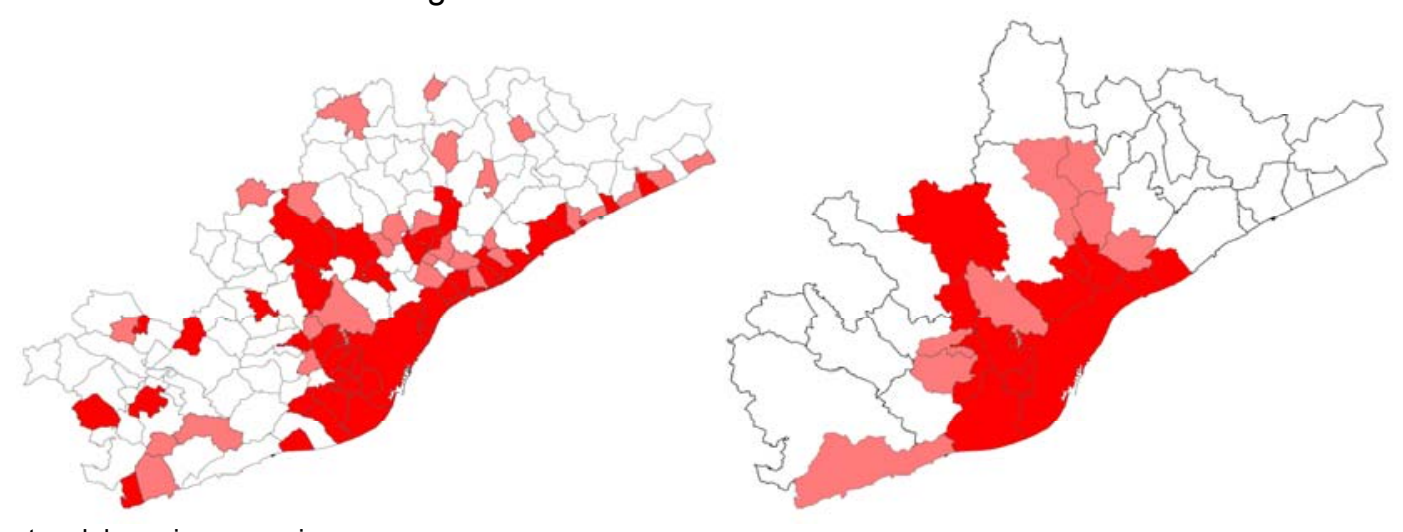

Fonte: elaborazione propria

Prendere coscienza di tali dinamiche permetterebbe di rivedere i "nuovi" confini di pianificazione e gli strumenti urbanistici più adatti, imponendo un cambio di scala a seconda della situazione contingente e particolari tipi di direttive da rispettare in fase progettuale, applicabili ad ambiti con caratteristiche similari di sviluppo.

L'uso di indicatori descrittivi per definire un tipo di forma urbana, potrebbe rappresentare, inoltre, un utile sostegno allo studio di modelli di città e territori sostenibili.

Sarebbe interessante poter "costruire" un modello di analisi delle tendenza di crescita urbana; stabilire in ambito costiero, ad esempio, una distanza massima alla quale l'azione plasmante del mare, sui fenomeni di trasformazione, comincia a perdere importanza; definire zone omogenee di pianificazione in cui tener conto di problematiche peculiari ed azioni settoriali specifiche a cui adattare gli interventi urbanistici.

\footnotetext{
${ }^{26}$ A livello territoriale, osservando la mappa dei protosistemi, si nota che nella parte nord-est la pressione di urbanizzazione si perde per effetto della fusione di municipi piccoli, ad alto tasso di urbanizzazione, con municipi più grandi in cui i livelli di pressione sono bassi. La mappa evidenzia maggiormente la forza centripeta di Barcellona.
} 
Anche nell'ambito di una stessa regione, ci sono zone che seguono canoni di sviluppo differenti dovuti a fattori di tipo geografico od economico: nei primi cinquanta chilometri, delle cinque regioni spagnole interessate dalla presenza del mediterraneo, la percentuale di urbanizzazione passa dal $50 \%$, a livelli molto bassi, di occupazione di suolo (Figura 13) decrescendo in maniera esponenziale: qual è l'effettivo peso della presenza del mare sulla crescita urbana e sui fenomeni economici ed in che dimensioni cambia al cambiare della distanza?

L'uso di modelli di analisi consentirebbe di "misurare" quanto, determinati fattori posizionali, la presenza di attrattori turistici ed economici e la disponibilità di costa "utile" influiscono sulle strutture urbane; trovare cioè quegli elementi che determinano l'insorgere dei fenomeni di espansione incontrollata più forti in determinati territori piuttosto che in altrí ${ }^{27}$.

\section{Figura 13. Suolo urbanizzato e distanza dal mare nelle regioni mediterranee spagnole ${ }^{28}$.}

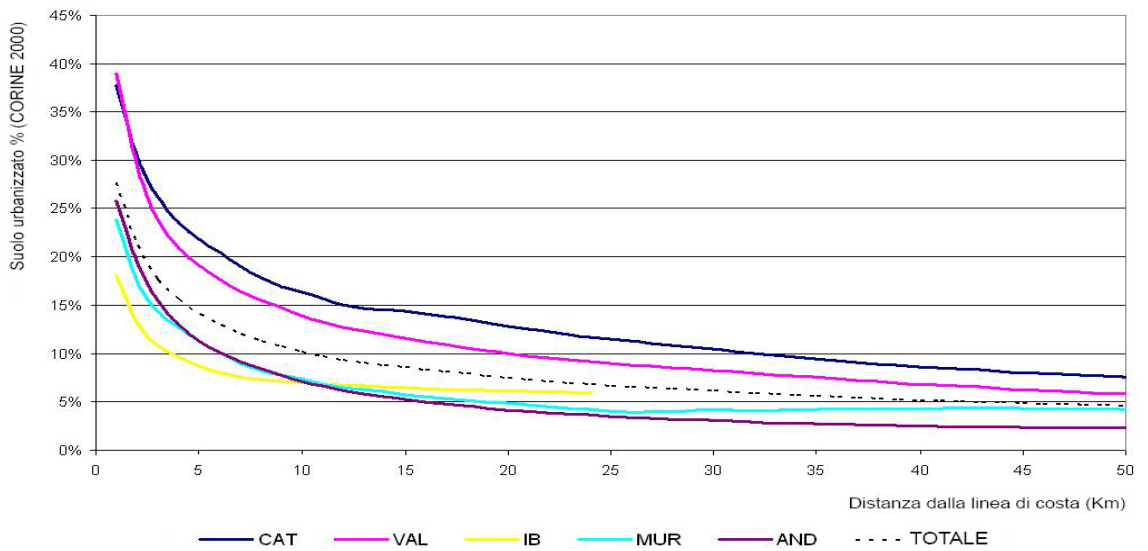

Fonte: workshop “El Proceso de Urbanización en la Costa Mediterránea Española” (Montserrat Moix Bergadá).

Stabilire la relazione tra $i$ vari attori che concorrono alla generazione di plusvalori nel mercato immobiliare ed il tipo di configurazione morfologica, "pesando" se, ed in che misura, l'influenza della costa agisce su tali valori e fino a che distanza dalla linea di costa sia possibile affermare che il mare co-partecipa alla formazione dei differenti gradi di complessità di specifici modelli di urbanizzazione.

\footnotetext{
${ }^{27}$ Lungo la costa mediterranea spagnola il $34 \%$ del primo $\mathrm{km}$ è urbanizzato e città come Melilla, Malaga e Barcellona elevano questa cifra a più del $50 \%$; in questa area le zone protette rappresentano solo un $5 \%$ del totale".

${ }^{28}$ Cataluña, Comunità Valenziana, Isole Baleari, Mursia, Andalusia.
} 


\section{Bibliografía.}

Anderson, John Ward. In Spain, a Tide Of Development. washingtonpost.com, 27.10.2006.

Baioni, Mauro. Diffusione, dispersione, anarchia urbanistica. Da: Antonio di Gennaro e Francesco P. Innamorato. La grande trasformazione. Il territorio rurale della Campania 1960/2000. Clean Edizioni, Napoli, luglio 2005 (pp. 82-87).

Barbaranelli, Claudio. Analisi dei dati con SPSS, II. Le analisi multivariate. LED Edizioni Universitarie di Lettere Economia Diritto, 2006.

Bergadá, Montserrat Moix. Workshop: El Proceso de Urbanización en la Costa Mediterránea Española. UPC, Barcelona, 14/03/2007.

Cappuccitti, Antonio. Morfogenesi dello spazio urbano: una lettura dei tessuti e delle misure, nella città di Roma. Berkeley.it, forme nella citta / spazi nella metropoli, urban forms and metropolitan spaces. Roma, 2-3 aprile 2007, Università degli Studi di Roma Sapienza, Facoltà di Ingegneria.

Cladera, Josep Roca; Bergadà, Montserrat Moix. Cap a una nova organització territorial de catalunya. Centre de Política de Sòl i Valoracions (CPSV). Barcelona, Maggio 2004.

Cori, Berardo. Scritti geografici in ricordo di Mario Ortolani - La pressione umana sulle aree costiere italiane. Roma, 1999.

Cori, Berardo. Med Picture - L'urbanizzazione costiera nel Mediterraneo. Genova, 2002.

Crotti, Sergio. "Determinazioni progettuali della morfogenesi urbana" in Metafora, mimesi, morfogenesi, progetto, (a cura di E.D'Alfonso e E.Franzini). Edizioni Angelo Guerini e Associati. Milano, 1991.

De Rossi, Domenico Alessandro. Sistema dei trasporti e sviluppo metropolitano, il caso pugliese. Mario Adda Editore. Bari, 2006.

Fabbri, Paolo. Nuove idee per la pianificazione costiera a fini turistico-ricreativi. Corso n. 2, Aspetti gestionali e socio-economici. Genova, 15 - 19 Giugno, 1998.

Novelli, Eugenio; Occelli, Sylvie. "Profili descrittivi di distribuzioni spaziali : alcune misure di diversificazione ». Cybergeo, Systèmes, Modélisation, Géostatistiques. Articolo 108, pubblicato il 4 ottobre 1999, modificato il 15 maggio 2007.

Secchi, Bernardo. Prima lezione di urbanistica. Edizioni Laterza. Bari, 2000.

Secchi, Bernardo. Tre storie per il ventesimo secolo. II International Ph.D Seminar on Urbanism. Barcelona, 27.06.2005.

Togna, Alessandro; Gravino, Salvatore. Rischio idraulico: Perimetrazione e classificazione delle aree a rischio di inondazione, Metodologie e linee guida per la prevenzione ed il controllo del rischio idrogeologico. A cura di: Ministero dell'Ambiente e della Tutela del Territorio. Direzione Generale Difesa Suolo, Progetto Operativo Difesa Suolo. Roma, 2006. 
